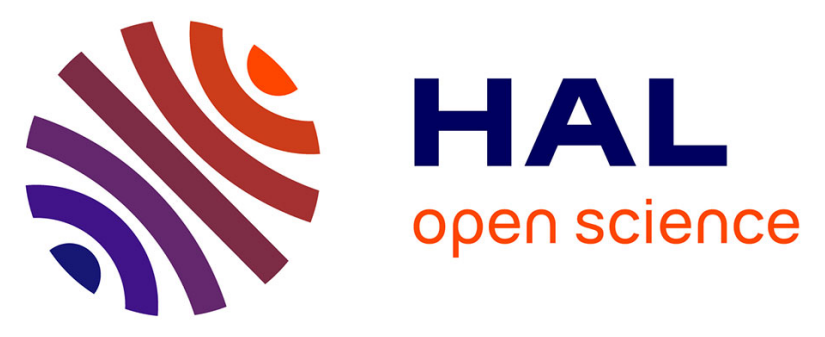

\title{
Robots for Learning - Learner-Centred Design
}

Wafa Johal, Barbara Bruno, Jennifer K Olsen, Mohamed Chetouani, Séverin

Lemaignan, Anara Sandygulova

\section{To cite this version:}

Wafa Johal, Barbara Bruno, Jennifer K Olsen, Mohamed Chetouani, Séverin Lemaignan, et al.. Robots for Learning - Learner-Centred Design. HRI '21: ACM/IEEE International Conference on Human-Robot Interaction, Mar 2021, Boulder CO, United States. pp.715-716, 10.1145/3434074.3444873. hal-03169202

\section{HAL Id: hal-03169202 https://hal.sorbonne-universite.fr/hal-03169202}

Submitted on 15 Mar 2021

HAL is a multi-disciplinary open access archive for the deposit and dissemination of scientific research documents, whether they are published or not. The documents may come from teaching and research institutions in France or abroad, or from public or private research centers.
L'archive ouverte pluridisciplinaire HAL, est destinée au dépôt et à la diffusion de documents scientifiques de niveau recherche, publiés ou non, émanant des établissements d'enseignement et de recherche français ou étrangers, des laboratoires publics ou privés. 


\section{Robots for Learning - Learner Centred Design}

\author{
Wafa Johal \\ UNSW Sydney \\ Sydney, Australia \\ wafa.johal@unsw.edu.au
Mohammed Chetouani
Sorbonne University \\ Paris, France \\ mohamed.chetouani@sorbonne- \\ universite.fr
}

\author{
Barbara Bruno \\ Swiss Federal Institute of Technology \\ in Lausanne (EPFL) \\ Lausanne, Switzerland \\ barbara.bruno@epfl.ch \\ Severin Lemaignan \\ Bristol Robotics Lab \\ Bristol, UK \\ severin.lemaignan@brl.ac.uk
}

\author{
Jennifer K. Olsen \\ University of San Diego \\ San Diego, USA \\ jenniferolsen@sandiego.edu
}

\author{
Anara Sandygulova \\ Nazarbayev University \\ Nur-Sultan, Kazakhstan \\ anara.sandygulova@nu.edu.kz
}

\begin{abstract}
The Robots for Learning workshop series aims at advancing the research topics related to the use of social robots in educational contexts. This year's half-day workshop follows on previous events in Human-Robot Interaction conferences focusing on efforts to discuss potential benchmarks in design, methodology and evaluation of new robotics systems that help learners. In this 6th edition of the workshop, we will be investigating in particular methods from technologies for education and online learning. Since the past few months, online and remote learning has been put in place in several countries to cope with the health and safety measures due to the Covid-19 pandemic. In this workshop, we aim to discuss strategies to design robotics system able to provide embodied assistance to the remote learners and to demonstrate long-term learning effects.
\end{abstract}

\section{CCS CONCEPTS}

- Human-centered computing $\rightarrow$ Human computer interaction (HCI); Interaction design; Empirical studies in HCI.

\section{KEYWORDS}

human-robot interaction, robots for learning

\section{ACM Reference Format:}

Wafa Johal, Barbara Bruno, Jennifer K. Olsen, Mohammed Chetouani, Severin Lemaignan, and Anara Sandygulova. 2021. Robots for Learning - Learner Centred Design. In Companion of the 2021 ACM/IEEE International Conferenceon Human-Robot Interaction (HRI'21 Companion), March 8-11, 2021,Boulder, CO, USA. ACM, New York, NY, USA, 2 pages. https://doi.org/10.1145/ 3434074.3444873

\section{OVERVIEW}

Imagine a person engaged in a learning activity, be it solving math exercises with pen and paper or practicing a second language by playing a memory game with a social robot. In both cases, the activity has superficial, visible goals (to find the solution of the

Permission to make digital or hard copies of part or all of this work for personal or classroom use is granted without fee provided that copies are not made or distributed for profit or commercial advantage and that copies bear this notice and the full citation on the first page. Copyrights for third-party components of this work must be honored

For all other uses, contact the owner/author(s).

HRI '21 Companion, March 8-11,2021, Boulder, CO, USA

(c) 2021 Copyright held by the owner/author(s).

ACM ISBN 978-1-4503-8290-8/21/03.

https://doi.org/10.1145/3434074.3444873 equation, to score more points than the other player) covering the deeper, more elusive goal of helping the person learn. In the specific case of learning activities involving social robot, this double-layered structure makes the design of the robot's behaviour a fascinating challenge: while it is relatively straightforward to design a robot that's a fierce competitor at a memory game, it is much less clear to envision how it should behave to support its human counterpart in learning. Similarly, the design of the experiment allowing for evaluating the robot's behaviour, as well as the robot-enhanced learning activity as a whole, becomes at once crucial and non trivial.

In this 6th edition of the "Robots for Learning" workshop, international experts in the field will share their knowledge concerning the design of social robot behaviours which support learning, their embedding in a learning activity and the evaluation of their impact through an experiment and the assessment of the person's learning. The workshop is organized as a highly interactive event, in which participants are encouraged to bring case studies taken from their own research and discuss them in groups and with the experts.

\subsection{Workshop Objectives}

The objectives of the workshops are to:

- Strengthen links between researchers in HRI working on Robots for Learning projects [1].

- Introduce and investigates methods from Educational Technologies in the Robots for Learning context (i.e. LearnerCentred Design, Learning Analytic [3]).

- Discuss benchmark in terms of design, methodology, learning scenario and reporting that could be adopted by the community to facilitate the inclusion of new researchers and the reproducibility of the results $[2,4]$.

\subsection{Format and Schedule}

We propose to organize a half day virtual workshop on the topic of Robots for Learning. The workshop will be online (e.g Zoom) and highly interactive. Participants will be asked to prepare the workshop by: 1) answering some questions related to their work and submit their answers; and 2) do some reading prior to the workshop. The workshop will have two types of session:

- Invited Speakers: invited senior researchers will share their perspectives and experiences on the field of technologies for 
education. They will be recorded to allow all participants watch them on-demand.

- Group Work Sessions: Each session aim to look at the participants' work with a certain angle, and will start with a short intro on the topic followed by a group work. Participants will be given tools to help them answer the questions relatively to their planned study/research.

We organized the different group sessions in 3 units:

Unit 1 Background and Research questions. During this session participants will exchange on their research objectives, research context and theoretical grounding of their work.

Unit 2 Design. This group work will aim to present and share about the learning context (i.e. defining the target learners, the learning objectives, the learning context).

Unit 3 Assessment. The last group session will focus on the metrics and measures that can be used in the context of robots for learning. Participants will discuss classical methods and share potential idea to use the robot's sensors to log interaction data to infer learning strategies.

\section{TARGET AUDIENCE}

We invite participants to report previous or planned research, practice and interest in developing applications in social robots for learning. Researchers from HRI, robotics and educational backgrounds will be invited to contribute. The workshop is an opportunity for researchers who want to further develop their research in the field of robots for learning to share and refine their ideas with peers.

The workshop will be advertised by sending a call-for-interest on robotics and technology for learning mailing lists and using social networks. Various projects involved in the organization will also be encouraged to participate (CoWriter, Cellulo, SAR NSF Expedition, eCUTE, DREAM, ANIMATAS, iReCheck ...). Previous editions of workshops on similar topics were held at RoMan 2016, HRI2017, HRI2018, HRI2019. The workshop will also be advertised to the people who attended the previous editions (a mailing list with about hundred contributors over the years has been constituted).

\section{ORGANISERS}

Wafa Johal, University of New South Wales, Sydney, Australia. Assistant Professor in the Faculty of Engineering at UNSW Sydney, Wafa obtained her $\mathrm{PhD}$ from the University of Grenoble (France) focusing on bodily signals in child-robot interaction and affective reasoning for social agents. Her research aims at creating acceptable and useful assistive robot interactions using social signal sensing, affective and cognitive reasoning and natural expressivity.

Barbara Bruno, Swiss Federal Institute of Technology in Lausanne (EPFL), Lausanne, Switzerland. Barbara is a post-doc researcher in the CHILI lab and BIOROB/MOBOTS group, actively interested in Social Robotics and Human-Robot Interaction and Cooperation. She received her Ph.D. in Robotics from the University of Genoa, Italy in 2015 and is currently involved in the EU ITN ANIMATAS, aiming at advancing intuitive human-machine interaction with human-like social capabilities for education in schools.

Jennifer K. Olsen, University of San Diego, USA. Jennifer is an assistant professor of computer science at the University of San Diego. She received her $\mathrm{PhD}$ from Carnegie Mellon University in human-computer interaction. Her research interests lie at the intersection of human-computer interaction, cognition, and education. Her current work focuses on how collaboration can support learning and how to design educational technology to support these practices from both the learners' and instructors' perspectives.

Mohamed Chetouani, Sorbonne University, Paris, France. Mohamed Chetouani is a Full Professor in signal processing and machine learning for human-machine interaction. His activities cover social signal processing, social robotics and interactive machine learning with applications in psychiatry, psychology, social neuroscience and education. Since 2018, he is the coordinator of the ANIMATAS H2020 Marie Sklodowska Curie European Training Network. He was the local co-chair of IEEE ICRA 2020 (Paris) and now the Program co-chair of ICMI 2020 (Utrecht). Since 2020, he is the Chair of the Ethical Advisory Board of Sorbonne University.

Séverin Lemaignan, Bristol Robotics Lab, Bristol, UK. Séverin is Associate Professor in Social Robotics and AI at the Bristol Robotics Lab. His research interest primarily concerns the socio-cognitive aspects of human-robot interaction, both from the perspective of the human cognition and the design of cognitive architectures for the robots. He has been focusing his recent experimental work on human-in-the-loop machine learning for social robots, with applications in child-robot interactions and assistive robotics, with the goal to develop effective and engaging novel interaction paradigms, in particular in education.

Anara Sandygulova, Nazarbayev University, Nur-Sultan, Kazakhstan. Anara Sandygulova is an Assistant Professor in the Department of Robotics and Mechatronics at the School of Science and Technology. She received her $\mathrm{PhD}$ in 2015 from University College Dublin where she investigated how children's perception of the robot changes with age and gender in order to make a robot adapt to these differences. Her research interests are human-oriented perception to enable adaptive human-robot interaction.

\section{ACKNOWLEDGMENTS}

Researchers organising this workshop would like to thank the following agencies for their funding support: the Australian Research Council (DE210100858), the Swiss National Science Foundation through the National Centre of Competence in Research Robotics, The European Union Horizon 2020 through the ANIMATAS project (No 765955) and HumanE AI NET project (No 761758), the Nazarbayev University Collaborative Research Program (No 091019CRP2107) and the Swiss State Secretariat for Education, Research and Innovation (SFG1038).

\section{REFERENCES}

[1] Robots for Learning Website. 2020. https://robot4learning.github.io/

[2] Wafa Johal. 2020. Research Trends in Social Robots for Learning. Current Robotics Reports (2020), 1-9.

[3] Zacharoula Papamitsiou and Anastasios A Economides. 2014. Learning analytics and educational data mining in practice: A systematic literature review of empirical evidence. Fournal of Educational Technology \& Society 17, 4 (2014), 49-64.

[4] Daniel B. Wright. 2019. Research Methods for Education With Technology: Four Concerns, Examples, and Recommendations. Frontiers in Education 4 (2019), 147. https://doi.org/10.3389/feduc.2019.00147 